\title{
Structures for Interacting Composite Fermions: Stripes, Bubbles, and Fractional Quantum Hall Effect
}

\author{
Seung-Yeop Lee, Vito W. Scarola, and J.K. Jain \\ Department of Physics, 104 Davey Laboratory, The Pennsylvania State University, University Park, Pennsylvania 16802
}

(April 15, 2002)

\begin{abstract}
Much of the present day qualitative phenomenology of the fractional quantum Hall effect can be understood by neglecting the interactions between composite fermions altogether. For example the fractional quantum Hall effect at $\nu=n /(2 p n \pm 1)$ corresponds to filled composite-fermion Landau levels, and the compressible state at $\nu=1 / 2 p$ to the Fermi sea of composite fermions. Away from these filling factors, the residual interactions between composite fermions will determine the nature of the ground state. In this article, a model is constructed for the residual interaction between composite fermions, and various possible states are considered in a variational approach. Our study suggests formation of composite-fermion stripes, bubble crystals, as well as fractional quantum Hall states for appropriate situations.
\end{abstract}

71.10.Pm,73.43.-f

\section{INTRODUCTION}

In two dimensions, electrons subjected to a strong magnetic field avoid one another by capturing an even number of quantized vortices and turning into composite fermions $[1,2]$. The composite fermions interact much more weakly than electrons. In fact, tremendous progress can be made towards understanding the dramatic behavior of this system by treating composite fermions as non-interacting. It is not that there is no interaction between the composite fermions, but it is weak and often does not alter the nature of the state in a qualitative manner, i.e., can be treated perturbatively. In such situations, while the inter-composite fermion ( $\mathrm{CF}$ ) interaction is surely important for quantitative considerations, it does not affect the qualitative phenomenology, and can be neglected altogether when the aim is to describe the qualitative phenomenology.

For example, the odd-denominator fractions $\nu=$ $n /(2 p n \pm 1)$ map into $\nu^{*}=n$ of composite fermions carrying $2 p$ vortices. Here, the system has a gap even in the absence of inter-CF interactions - in stark contrast to the electron system at $\nu=n /(2 p n \pm 1)$ which had an enormous degeneracy in the absence of interactions - and it is possible that for many of these filling factors, the gap would not disappear as the inter-CF interaction is slowly turned on to its physical value. If that were the case, the inter-CF interaction is unimportant at a qualitative level. This explains the fractional quantum Hall effect [3] (FQHE) at filling factors $\nu=n /(2 p n \pm 1)$ in terms of the integral QHE [4] of composite fermions [1].

Another example is at fractions $\nu=1 / 2 p$, which map into composite fermions at zero effective magnetic field. If the interactions between composite fermion are neglected, a Fermi sea is obtained [5]. Conceptually, it is more difficult to justify the neglect of inter-CF interac- tions in this case, as there is no gap for the Fermi sea. Nonetheless, at least there is a well defined ground state for composite fermions, unlike for non-interacting electrons at $\nu=1 / 2 p$, and various experimental studies [6] have shown that the CF Fermi sea is a good starting point for many purposes.

We recall that at relatively high temperatures and low mobilities only the integral quantum Hall effect was seen, which is explained by neglecting the interaction between electrons. As the sample quality improved and temperature lowered, FQHE was observed, which is understood in terms of weakly interacting composite fermions. While the model in which the composite fermions are taken as non-interacting has been strikingly successful, one may ask if another set of new structures would emerge as the experiments further improve. This question has motivated our investigation of the subtle physics arising from inter-CF interactions. Fortunately, there already exists a good microscopic approach for investigating this issue, in the form of the wave functions for composite fermions [1]. Even though these wave functions are motivated by the physics of independent composite fermions, they give an excellent description of the interaction between the composite fermions $[7,8,2]$. In fact, the wave functions practically give the exact solution to the problem, which implies that they incorporate the full interaction effects. For example, consider the low-energy branch of excitations, described as an exciton of composite fermions. If the composite fermions were non-interacting, this branch would not have any dispersion. In reality, there are oscillations in the energy as a function of the wave vector, arising from the residual interaction between the $\mathrm{CF}$ particle and the CF-hole. The dispersion computed from the wave functions provides an accurate account of the oscillations. The wave functions similarly obtain the energy of two CF particles or two CF holes quite accurately. 
The inter-CF interactions are of course always relevant to quantitative issues. However, there are two situations in which they can also make a qualitative difference in the physics. The first situation is when the inter-CF interaction overwhelms the gap of the non-interacting $\mathrm{CF}$ model, thereby destroying the FQHE $[9,10]$. It has been shown that for $\nu \leq 1 / 9$ as well as for most fractions in higher Landau levels, the FQHE undergoes an excitonic instability, i.e., the energy of the exciton becomes smaller than the energy of the uniform filled CF-Landau level (LL) state. The CF theory thus not only tells us where FQHE occurs but also where it does not. (Recent experiments [11] have shown evidence for FQHE at $1 / 9$ at finite temperatures. Even though this observation is not presently understood, it ought to be stressed that it is not inconsistent with the earlier theoretical predictions which only address the zero temperature phase.) A pairing instability has also been suggested at $\nu=5 / 2$, i.e., at the half filled second LL, where the residual interaction between the composite fermions is attractive $[12,13]$. The resulting instability of the CF Fermi sea causes a gap to open up here, producing a plausible scenario for the $5 / 2$ fractional quantum Hall effect [14].

The other situation where the inter-CF interaction is expected to be important is away from the special filling fractions $\nu=n /(2 p n \pm 1)$, where the topmost CF-LL is only partially occupied, and the ground state has a large degeneracy if the inter-CF interaction were to be turned off. The degeneracy for independent composite fermions is much smaller than for independent electrons even here, making the former a better defined starting point. The weak residual interaction between the composite fermions will decide the nature of the true ground state here. The present article deals with such situations.

The first possibility that comes to mind when composite fermions partially occupy a Landau level is that of their fractional QHE. This could happen when the composite fermion filling factor is $\nu^{*}=n \pm \bar{n} /(2 \bar{p} \bar{n} \pm 1)$, which correspond, as usual, to electrons filling factors given by $\nu=\nu^{*} /\left(2 p \nu^{*} \pm 1\right)$. We will consider the special case when the base composite fermions are carrying two vortices, denoted by ${ }^{2} \mathrm{CFs}$, and the ${ }^{2} \mathrm{CFs}$ in the topmost partially occupied CF-LL capture 2 additional vortices to become ${ }^{4} \mathrm{CFs}$ (composite fermions carrying four vortices) and condense into an incompressible state with $\bar{n}$ filled Landau levels. The full state is then made up of two flavors of composite fermions, with ${ }^{2} \mathrm{CFs}$ in the lower filled CF LLs, and ${ }^{4} \mathrm{CFs}$ in the topmost CF-LL. Such states are quite analogous to the FQHE state at $\nu=2+1 / 3$, where electrons in the second LL convert into composite fermions whereas the electrons in the lowest LL remain electrons. Such states have been considered in the past $[1,15,16]$, dubbed mixed flavor states of composite fermions. Of course, depending on the filling, it is also possible that composite fermions would capture additional vortices to form a Fermi sea or a paired state.
Yet another possibility is for them to not capture any new vortices but rather to phase separate in some manner, possibly through formation of stripes. Our aim in this article is to consider these possibilities as quantitatively as presently possible over a range of filling factors.

It should be emphasized that while these states are easily viewed in terms of composite fermions, they are fantastically complicated in terms of electrons. Consider the possibility of pairing at $\nu=3 / 8$, for example [16]. First, all electrons capture vortices to turn into ${ }^{2} \mathrm{CFs}$ at $\nu^{*}=1+1 / 2$; then, those in the half filled CF-LL put on two more vortices, and attempt to make a ${ }^{4} \mathrm{CF}$ Fermi sea; but the Fermi sea is unstable to pairing; pairing opens up a gap to produce fractional quantum Hall effect. Such a state could perhaps not be envisioned without the knowledge of composite fermions.

In an earlier short article [17], we had studied composite fermion fillings given by $\nu^{*}=n+1 / 2$ by this method. The present article gives further details and also considers a broader range of filling factors. The plan of the article is as follows. In the next section, we enumerate the various approximations that go into the calculation. Sect. III discusses how we obtain the pseudopotentials for the inter-CF interaction, and how we obtain from it a real space interaction between composite fermions. Certain preliminary observations are made in Section IV. The variational wave functions are given in Section V, and Section VI discusses the results of our calculations. In Section VII, we briefly investigate the effect of finite thickness on our results.

\section{ASSUMPTIONS}

We will be studying composite fermions at fillings of the type $\nu^{*}=n+\nu_{n}^{*}$, where $n$ is the number of filled composite fermion Landau levels and $\nu_{n}^{*}$ is the filling fraction of the topmost composite fermion Landau level. In order to make progress, we will make several assumptions.

The system will be taken to be fully spin polarized, which is a valid approximation for sufficiently high magnetic fields. The method can be adapted to situations for which the states are only partially spin polarized, [16] but here we will confine our attention to fully spin polarized systems.

We will map below the problem of interacting electrons into that of interacting composite fermions only of the topmost partially filled CF Landau level. The interaction between composite fermions will be obtained by "integrating out" the composite fermions of the lower filled CF-LLs.

We will assume that the filled CF Landau levels can be treated as inert and the problem can be defined solely in terms of the composite fermions in the topmost partially filled CF Landau levels. This should be qualitatively correct between well defined FQHE states described by 
filled CF Landau levels. It should also be a quantitatively accurate approximation when the CF-LL separation is large compared to the inter-CF interaction. Indeed, for filling factor in the range $1 / 3<\nu<2 / 5$ the composite fermion theory neglecting CF-LL mixing provides a very good description of the actual states obtained from exact diagonalization $[7,8]$. However, this approximation may become worse when the effective cyclotron energy is not too large; here the hybridization of $\mathrm{CF}$ states with higher CF Landau levels may become relevant.

Given that the composite fermion system actually represents a strongly correlated state of matter, the interaction energy for composite fermions in general involves two-, three-, and $n$-body terms. The most critical assumption will be our neglect of three and higher body terms. Following Wojs and Quinn [18] we will assume that the interaction energy of the many composite fermion system can be modeled as a sum of pair-wise interaction. Furthermore, we will determine the pair interaction from the system that contains only two composite fermions in the partially occupied CF Landau level (with the lower CF Landau levels fully occupied). This assumption is valid only if the three and higher body terms do not cause a phase transition.

There are indications that the higher order terms are small. The exact energy spectrum of the system with three composite fermions in the second CF-LL (where the only input is the Coulomb interaction between the electrons) has been compared with the energy spectrum that the pair-wise interaction predicts, and a reasonably good agreement is obtained [18]. The latter spectrum is obtained by diagonalizing the effective two-body interaction between composite fermions obtained by the method outlined earlier. However, no systematic study of the effects of multi-particle interaction has been performed as a function of the number of particles.

Another limitation of our work is its variational nature, which makes it as good as the trial wave functions for various states. For example, for FQHE states, we will use the standard wave functions for composite fermions [1]. These are extremely accurate in the lowest Landau level, but not so accurate for FQHE in higher Landau levels. Of course, it is also possible that the actual ground state has some completely new structure, not described physically by any of the trial states considered here.

These caveats are meant to stress right at the outset that the results below are not "hard" predictions of the composite fermion theory, but are predicated upon several assumptions. This study is the first step toward a theoretical treatment of the interaction between composite fermions, and it is hoped that future experimental and theoretical studies will shed light on the reliability of our present model.

\section{MODEL FOR INTERACTION BETWEEN COMPOSITE FERMIONS}

\section{A. Pseudopotentials for CF-CF interaction}

It is known that a two-body interaction of fermions confined to some Landau level is fully characterized by its Haldane pseudopotentials [19], $V_{m}$, which are the energies of two particles in the relative angular momentum state $m,\left|\psi_{m}\right\rangle$ :

$$
\begin{aligned}
V_{m} & =\frac{\left\langle\psi_{m}|V| \psi_{m}\right\rangle}{\left\langle\psi_{m} \mid \psi_{m}\right\rangle} \\
& =\frac{1}{2^{2 m+1} m ! l_{0}^{2 m+2}} \int r d r V(r) r^{2 m} e^{-r^{2} / 4 l_{0}^{2}} \\
& =\int q d q \tilde{V}(q) e^{-q^{2} l_{0}^{2}} L_{m}\left(q^{2} l_{0}^{2}\right)
\end{aligned}
$$

Here $l_{0}$ is the magnetic length, $L_{m}$ is a Laguerre polynomial and $\left|\psi_{m}\right\rangle$ is the two-body state of relative angular momentum $m$. For fully spin polarized fermions, only the pseudopotentials $V_{m}$ with odd integer values of $m$ are relevant. In this paper, the Fourier transformation is defined symmetrically

$$
\tilde{V}(q) \equiv \frac{1}{2 \pi} \int d^{2} r V(r) e^{-i \vec{q} \cdot \vec{r}}=\int r d r J_{0}(q r) V(r)
$$

where $J_{0}$ is a Bessel function. In the equations below, the interelectron interaction, $V(r)=e^{2} / \epsilon r$, will be expressed in units of $e^{2} / \epsilon l_{0}$, and $r$ in units of $l_{0}$; the interelectron interaction then becomes $V(r)=1 / r$. The appropriate units will be put back at the end.

Given the pseudoptentials we can construct the two body interaction in the lowest LL using the following formula.

$$
\tilde{V}(q)=2 \pi \sum_{m} V_{m} L_{m}\left(q^{2}\right)
$$

Alternatively, we can directly write the Hamiltonian as

$$
H|\psi\rangle=\sum_{i<j} \sum_{m} V_{m} P_{m}^{i j}|\psi\rangle=\sum_{i<j} \sum_{m=o d d} V_{m} P_{m}^{i j}|\psi\rangle .
$$

Here $P_{m}^{i j}$ projects particles $i$ and $j$ onto a state of relative angular momentum $m$. This Hamiltonian is useful for exact diagonalization study.

The pesudopotentials for composite fermions are defined similarly. For the pseudopotentials in the $n$th composite fermion Landau level, we consider the state in which the lower CF Landau levels are fully occupied and the $(n+1)^{s t} \mathrm{CF}$ Landau levels contains two composite fermions. $V_{m}^{C F}$ is then defined as the energy of the state in which the two composite fermions are in the relative angular momentum $m$ state. In other words, 


$$
V_{m}^{C F}=\frac{\left\langle\psi_{m}^{C F}\left|\sum_{i<j} \frac{1}{r_{i j}}\right| \psi_{m}^{C F}\right\rangle}{\left\langle\psi_{m}^{C F} \mid \psi_{m}^{C F}\right\rangle}-E_{\infty}
$$

where $\left|\psi_{m}^{C F}\right\rangle$ is the wave function of composite fermions in the relative angular momentum $m$ states. Note that the state of two composite fermions in the $(n+1)$ st level is a many body state of electrons. For convenience, the energy is measured relative to $E_{\infty}$, the energy of the state in which the two composite fermions are far separated. (For finite systems the value of $E_{\infty}$ is chosen in the manner explained below.)

We use the spherical geometry for our calculations. [19] Following the standard procedure for writing the wave functions for composite fermions [1] the wave function for the relevant state with angular momentum $L$ is given by

$$
\left|\psi_{L}^{C F}\right\rangle=\mathcal{P}_{L L L} \Phi_{1}^{2} \Phi_{n}^{++L},
$$

where $\Phi_{1}$ is the wave function of one filled LL, $\Phi_{n}^{++L}$ is the wave function of the electron state in which $n$ LLs are fully occupied and the $(n+1)$ st LL contains two electrons in angular momentum $L$ state:

$$
\Phi_{n}^{++L}=\sum_{m_{1}, m_{2}}<L M \mid l m_{1} ; l m_{2}>a_{n+1, m_{1}}^{+} a_{n+1, m_{2}}^{+} \Phi_{n}
$$

Here $\Phi_{n}$ is the state with n-filled LLs, $a_{n+1, m_{1}}^{+}$creates an electron in the $l_{z}=m_{1}$ state in the $(n+1)$ th LL, $l$ is the angular momentum of a single composite fermion in the $(n+1)^{s t} \mathrm{LL}$, and $<L M \mid l m_{1} ; l m_{2}>$ are ClebschGordon coefficients. Because the energy depends only on the total angular momentum $L$, we work with $M=0$, i.e., $m_{1}+m_{2}=0$. The explicit form for the general wave functions of this kind is given in the literature [8]. $\mathcal{P}_{L L L}$ is the lowest LL projection operator.

In the spherical geometry, $V_{m}$ is defined as the energy of the state with angular momentum $L=2 l-m$ [20]:

$$
V_{m}^{C F}=\frac{\left\langle\psi_{2 l-m}^{C F}\left|\sum_{i j} \frac{1}{r_{i<j}}\right| \psi_{2 l-m}^{C F}\right\rangle}{\left\langle\psi_{2 l-m}^{C F} \mid \psi_{2 l-m}^{C F}\right\rangle}-E_{\infty}
$$

This brings the definition of the pseudopotentials into conformity with the disk geometry, reproducing the disk pseudopotentials in the thermodynamic limit $N \rightarrow \infty$, when the angular momentum of the lowest Landau level shell also diverges: $l \rightarrow \infty$. To see this, it is helpful to note that in the disk geometry, the separation between the two particles increases with $m$. In the spherical geometry, the smallest separation is obtained when both particles are put in the same $l_{z}$ state, which corresponds to the largest angular momentum $L=2 l$ (not bothering about the Pauli principle here). Thus, the largest $L$ corresponds to the smallest $m$.

The integrals are performed using Monte Carlo; we evaluate $V_{m}^{C F}$ for up to 50 particles. Of particular interest is the short range part of the interaction, which appears to converge to the thermodynamic limit fairly quickly with the number of particles, as expected. The long range part of the interaction is explicitly known, as discussed below.

In order to minimize the computation time, we calculate several pseudopotentials within one Monte Carlo run by sampling with one wave function $\psi_{m_{s}}^{C F}(\mathbf{r})$, according to

$$
\begin{aligned}
V_{m}^{C F} & \stackrel{*}{=} \int d \mathbf{r}\left|\psi_{m}^{C F}(\mathbf{r})\right|^{2} \sum_{i<j} \frac{1}{r_{i j}} \\
& =\int d \mathbf{r}\left(\frac{\left|\psi_{m}^{C F}(\mathbf{r})\right|^{2}}{\left|\psi_{m_{s}}^{C F}(\mathbf{r})\right|^{2}} \sum_{i<j} \frac{1}{r_{i j}}\right)\left|\psi_{m_{s}}^{C F}(\mathbf{r})\right|^{2}
\end{aligned}
$$

where $(\stackrel{*}{=})$ means that the two sides are equal upto an additive constant. Generally, the error increases as the angular momentum moves away from the sampling angular momentum, and one must eventually use a different state for sampling; however, sampling with a single state works satisfactory for $N \leq 40$, where $N$ is the total number of particles.

In Monte Carlo with composite fermions, all elements of the determinant are altered even if a single particle is moved, because of the strongly correlated nature of the problem. However, in a given step, the determinants that combine to give the state with a well defined $L$ differ only in two columns. Rather than calculating each determinant entirely, we use an updating trick earlier used in Ref. [21], which is a generalization of a method used for updating determinants that differ only in one column [22]. The method is explained in the Appendix.

The pseudopotentials for the CF-CF interaction are shown in Fig. 1 of Ref. [17] and Fig (1). The explicit values are given in Tables I-VI.

\section{B. Inter-CF interaction in real space}

Now that the composite fermions of the fully occupied CF-LLs have been integrated out to produce an effective interaction between the composite fermions of the partially filled CF-LL, only the $N$ composite fermions in the topmost partially filled LL will be explicitly considered in what follows. (Earlier, the symbol $N$ was used for the total number of composite fermions, but below it will be reserved for the number of composite fermions in the partially filled CF-LL.)

We thus have a system of fermions restricted to a certain CF LL, with an interaction known through its pseudopotentials. It would be most natural to feed the pseudopotentials into an exact diagonalization routine and compute the ground state. However, such studies are not useful for some of the states that we wish to consider. The system sizes accessible to exact diagonalization are too small to capture the physics of compressible or stripe 
states. (Also, the spherical geometry often used for exact diagonalization is not particularly convenient for the stripe phase.)

We shall instead proceed by considering variational wave functions for several different kinds of states, and determining which has the lowest energy. As mentioned earlier, this approach has the shortcoming that we do not know if we have missed the true ground state, and even for a given state, we may not have a sufficiently accurate wave function. We will consider all of the states that have been investigated in the past in the lowest and higher Landau levels of electrons, and hope that the actual ground state is one of them. Ultimately, only experiments can tell us for sure if that is the case.

We will evaluate the energies of the some of the trial wave functions using the Monte Carlo method, for which we will need a real space form for the interaction between composite fermions. Furthermore, it is convenient to write various trial wave functions in the lowest Landau level. So, we will map the problem of composite fermions at $\nu^{*}=n \pm \nu_{n}^{*}$ into that of $N$ fermions in the lowest LL at $\nu_{n}^{*}$. Such a mapping can, in principle, be carried out exactly, because the problem of fermions in any given LL with one interaction is equivalent to that of fermions in a different LL with another interaction, provided the Haldane pseudopotentials for the two interactions are the same. Defining the problem in the lowest LL helps us to find the energies for various composite fermion states, because convenient techniques exist for writing the wave functions in explicit form in the lowest LL.

For some of the states (paired state, Fermi sea) the calculation will be done in the spherical geometry, and the energy in the thermodynamic limit $(N \rightarrow \infty)$ will be estimated by an extrapolation of finite system results. For the stripe and bubble phases, the calculations will be done directly for the thermodynamic limit in the planar geometry. In this case, the spherical geometry is used only for the determination of the effective interaction between composite fermions.

In order to map the problem of composite fermions at $\nu^{*}=n \pm \nu_{n}^{*}$ into fermions at $\nu_{n}^{*}$ in the lowest Landau level, we need to find an effective interaction $V_{e f f}(r)$ which produces the desired pseudopotentials in the lowest Landau level. A proper consideration of the long range part of the interaction is important for our purposes below, especially in the context of stripes, the period of which is determined by a competition between the short range attraction and long range repulsion. Therefore, we treat the long distance part of the interaction explicitly in our calculations. Fortunately, the form of the interaction in this limit is known: it is given by the Coulomb interaction between particles of fractional charge $e /(2 n+1)$, i.e.,

$$
\frac{(2 n+1)^{-2}}{r}\left[\frac{e^{2}}{\epsilon l_{0}}\right]
$$

where $r$ is the dimensionless distance between the composite fermions measured in units of $l_{0}$, the actual magnetic length at $\nu$. At the effective magnetic field, i.e., the magnetic field corresponding to the filling factor $\nu^{*}$, the natural unit for length is $l^{*}$. We denote the distance measured in units of $l^{*}$ by $r^{*}$. The long range part then translates into:

$$
\frac{(2 n+1)^{-2}}{r^{*}} \frac{l_{0}}{l^{*}}\left[\frac{e^{2}}{\epsilon l_{0}}\right] \rightarrow \frac{(2 n+1)^{-5 / 2}}{r^{*}}\left[\frac{e^{2}}{\epsilon l_{0}}\right]
$$

where we have used $l_{0} / l^{*}=(2 n+1)^{-1 / 2}$. We model the full interaction between composite fermions at the effective magnetic field $B^{*}$ by the following form:

$$
V^{e f f}\left(r^{*}\right)=\left(\sum_{i} c_{i} r^{* 2 i} e^{-r^{* 2}}+\frac{(2 n+1)^{-5 / 2}}{r^{*}}\right)\left[\frac{e^{2}}{\epsilon l_{0}}\right]
$$

The power series, with properly chosen coefficients $c_{i}$, takes care of the short range part of the inter-CF interaction. The functions of the kind $r^{* 2 i} e^{-r^{* 2}}$ are used because the pseudopotentials for such functions are analytically calculable. Many other functional choices could work equally well; the mapping from pseudopotentials to a real space interaction in the lowest LL is one-to-many, and all real space interactions with the same pseudopotentials are completely equivalent in the absence of $\mathrm{LL}$ mixing. (Slightly different functions were chosen in Ref. [23]. There, the long rage Coulomb interaction was not considered explicitly, because that work dealt with the composite fermion Fermi sea, which corresponds to the limit $n \rightarrow \infty$, so the last terms vanishes.) It is important to choose a real space form for the effective interaction that makes the interaction as smooth as possible; large oscillations of the interaction lead to larger errors in the Monte Carlo evaluation of the energy.

The units for the effective potential defined above ought to be noted. If we replace $e^{2} / \epsilon l_{0}$ by $e^{2} / \epsilon l^{*}$, then the interaction has the natural form for a problem at the effective magnetic field. We do our calculations with this interaction potential, and then obtain the final energies by a change of units.

Next, we proceed to find values for the coefficients $c_{i}$ so that the pseudopotentials of $V^{e f f}$ reproduce the pseudopotentials $V_{m}^{C F}$ calculated earlier from microscopic wave functions. Because we are considering fully polarized states, only the odd pseudopotentials are relevant. In principle, it is possible to fix all of the $c_{i}$ 's to reproduce all $V_{m}^{C F}$ 's exactly, but in practice, we will fix the first several (five to six) odd pseudopotentials only. That is sufficient for our purposes, given many other approximations that go into the calculation. The energy is determined predominantly by the short distance part of the interaction. Of course, at long distances, the above model interaction automatically gives the correct behavior, thereby ensuring that it is quite accurate almost everywhere. 
The energy of two composite fermion particles evaluated from microscopic wave functions gets contribution from two sources, their self energies and their interaction. The self energy can be identified by putting them far apart; because it is constant, it does not affect the calculation in any way. We find it convenient to subtract it out by shifting all $V_{m}^{C F}$ by a constant so that the last pseudopotential (remember that there are only a finite number of pseudopotentials in a finite system) becomes equal to the pseudopotential of the long range part of the model interaction:

$$
E_{\infty}=\frac{\left\langle\psi_{m_{\max }}\left|\frac{(2 n+1)^{-\frac{5}{2}}}{r}\right| \psi_{m_{\max }}\right\rangle}{\left\langle\psi_{m_{\max }} \mid \psi_{m_{\max }}\right\rangle}
$$

where $\left|\psi_{m_{\max }}\right\rangle$ is the two-body state with relative angular momentum $m$ in the LLL. It is expected that for sufficiently large systems, the contribution of the gaussian terms to the last pseudopotential is negligible due to their rapid decrease with distance.

We have matched the first five or six odd pseudopotentials by adjusting the same number of coefficients $c_{i}$. The values of $c_{i}$ used below are quoted in Tables (VII) and (VIII). The comparison between the pseudopotentials $V_{m}^{C F}$, calculated from wave functions, and the pseudopotentials calculated from the real space model interaction are shown in Fig. 1 of Ref. [17] and 1, indicating that the real space interaction provides a good representation of the $V_{m}^{C F}$. The specific choice of which $c_{i}$ are taken to be non-zero in our work (Tables VII and VIII) is dictated by the smoothness of the real space form of the interaction. We also note that the form of $V^{e f f}\left(r^{*}\right)$ depends on details, and can appear rather strange, with oscillations and even attractive regions, but proper results are guaranteed so long as it generates the correct pseudopotentials.

\section{PRELIMINARY OBSERVATIONS}

A glance at the CF pseudopotentials reveals one of the most striking features of the inter-CF interaction: it is often attractive. The smallest energy is obtained at the smallest relative angular momentum, i.e., at the shortest distance. This is true for CF particles in the third or higher CF-Landau levels and for CF holes in the second and higher CF-LLs. This fact already appears to suggest an answer to one important question: Are there any new FQHE states between the fractions $n /(2 n+1)$ ? New FQHE states here can appear if ${ }^{2} \mathrm{CFs}$ in a partially filled CF-LL capture additional vortices to form a FQHE state. However, capture of vortices requires a sufficiently strong short range repulsion. Given that the inter-CF interaction is actually attractive, any new FQHE in the range $2 / 5<\nu<1 / 2$ appears unlikely (for fully polarized systems), to the extent that our model for interacting composite fermions is trustworthy. (The caveat is important; for example, three body interaction could play an important role, or when there are many composite fermions, the nature of the two-body interaction might change slightly from our model which considers only two composite fermion particles.) Nonetheless, the form of the interaction suggests that new FQHE states in the range $2 / 5<\nu<1 / 2$ are either very weak or altogether absent. That is entirely consistent with experiments.

For composite fermions in the lowest CF Landau level $(\nu<1 / 3)$, on the other hand, the interaction is strongly repulsive (Fig. 1, top panel), and new FQHE is expected. That is also consistent with experiment. The ${ }^{2} \mathrm{CFs}$ capture two additional flux quanta to become ${ }^{4} \mathrm{CFs}$, which then fill Landau levels to produce FQHE at $\nu=$ $n /(4 n \pm 1)$. There is presently experimental evidence for six members of these sequences [11]. These states can be interpreted either as the IQHE of ${ }^{4} \mathrm{CFs}$ or as the FQHE of ${ }^{2} \mathrm{CFs}$; they are "pure," (as opposed to "mixed") in the sense that they contain only a single flavor of composite fermions.

The CF pseudopotentials in Fig. 1 of Ref. [17] and Fig. (1) of this paper also indicate that the assumption of treating the interaction between many composite fermions as the sum of two body interactions is not unreasonable. In a given CF-LL, Fig. 1 of Ref. [17] gives the interaction between two $\mathrm{CF}$ particles in an otherwise empty CF-LL, whereas Fig. 1 gives the interaction between two CF holes in an otherwise full CF-LL. The latter is a many body state of CF particles. However, the two interactions are rather similar both in shape as well as magnitude, which is what would be expected if only two body terms were relevant.

\section{VARIATIONAL STATES}

\section{A. ${ }^{4}$ CF FQHE / ${ }^{4}$ CF Fermi sea}

One possibility for composite fermions in the partially filled ${ }^{2} \mathrm{CF}$-LL is to capture two additional vortices to turn into ${ }^{4} \mathrm{CFs}$ and then form an integral quantum Hall state with $n^{\prime}$ filled Landau levels, which corresponds to

$$
\nu^{*}=n \pm \frac{n^{\prime}}{2 n^{\prime} \pm 1}
$$

filling for ${ }^{2} \mathrm{CFs}$, and to

$$
\nu=\frac{\nu^{*}}{2 \nu^{*} \pm 1}
$$

for electrons.

Taking ${ }^{2} \mathrm{CFs}$ as base particles, the wave function for their FQHE state is given by:

$$
\Psi_{\frac{n^{\prime}}{2 n^{\prime}+1}}^{F Q H E}=P_{L L L} \Phi_{1}^{2 p} \Phi_{n^{\prime}}
$$


where $\Phi_{n^{\prime}}$ is the wave function of $n$ fully filled LLs and $P_{L L L}$ projects the state onto the lowest LL. Recall only the ${ }^{2} \mathrm{CFs}$ in the partially filled CF-LL are considered explicitly here; the filled ${ }^{2} \mathrm{CF}$ Landau levels have been integrated out in producing the effective interaction. The state at $\nu^{*}=n+1 / 2$ is obtained in the limit $n^{\prime} \rightarrow \infty$, where the filled Landau level state $\Phi_{n^{\prime}}$ becomes a Fermi sea. We expect these states to be energetically favorable when $V_{1}^{C F}$ is large compared to other pseudopotentials.

The energies of these states can be calculated with the help of the real space interaction, following the method outlined in the literature. The spherical geometry will be used in our calculations, and the thermodynamic energy will be obtained from a linear, least squares fit of the finite system energies plotted as a function of $1 / N$.

\section{B. ${ }^{4} \mathrm{CF}$ pairing}

At half filled Landau level, pairing of composite fermions also becomes possible. A weak repulsion between fermions is believed to lead to pairing of composite fermions at half filling $[12,13,16,23]$. The second LL coulomb interaction between electrons falls within this class of interactions. The paired state is reasonably well described by a Pfaffian wave function [13], which, in particular, has been shown to have lower energy than the $\mathrm{CF}$ Fermi sea at $\nu=5 / 2$ [23]. Numerical studies on small systems have shown that this state has a fairly large overlap with the exact ground state [16], although the overlaps are not decisive (in contract to the case for filled composite fermion Landau levels for which the overlaps are in excess of 99\%).

We ask whether or not the interaction between CFs at half filled CF LLs favors the paired state of ${ }^{2} \mathrm{CFs}$. Here, ${ }^{2} \mathrm{CFs}$ capture two vortices to turn into ${ }^{4} \mathrm{CFs}$ and then form pairs. With ${ }^{2} \mathrm{CFs}$ as base particles at filling factor equal to $1 / 2$, we consider the Pfaffian wave function for the paired state:

$$
\Psi_{\frac{1}{2}}^{P f}=\Phi_{1}^{2} \operatorname{Pf}[M]
$$

where $\operatorname{Pf}[M]$ is the Pfaffian of the matrix $M$ with components $M_{i j}=\left(z_{i}-z_{j}\right)^{-1}$ defined as $\operatorname{Pf}[M] \propto$ $A\left[M_{12} M_{34} \ldots\right]$. Pf $[M]$ is a real space BCS wave function and so $\Psi_{\frac{1}{2}}^{P f}$ can be viewed as a $\mathrm{p}$-wave paired quantum Hall state of ${ }^{4} \mathrm{CFs}$. We calculate the energy of this state as discussed in the previous section.

\section{C. ${ }^{2}$ CF stripes and bubble crystals}

For an attractive interaction, phase separation is a likely possibility, which, from our experience with higher Landau level physics, would lead to the formation of stripes or bubble crystals. In this case, the base particles, ${ }^{2} \mathrm{CF}$, do not capture any additional vortices. We calculate the energies of these states closely following the Hartree-Fock formulation used earlier [24,25] for electrons in higher Landau levels; the only difference is the form of the interaction. We give here a brief outline of the calculation for completeness, which closely follows Ref. [24].

Consider fermions in the plane of size $L_{x} \times L_{y}$. In the lowest LL the eigenstates are

$$
\psi_{k}(x, y)=\frac{1}{\sqrt{L_{y} l_{0} \pi^{1 / 2}}} e^{i k y} e^{-\frac{1}{2 l_{0}^{2}}\left(x+k l_{0}^{2}\right)^{2}}
$$

where $k \in \frac{2 \pi}{L_{y}} \times$ (integer). Define the operator $\Psi(\mathbf{r}) \equiv$ $\sum_{k} \psi_{k}(\mathbf{r}) a_{k}$ where $a_{k}$ is the annihilation operator corresponding to the state $\left|\psi_{k}\right\rangle$. With the Fourier transform defined as

$$
\tilde{f}(\mathbf{q})=\frac{1}{2 \pi} \int d^{2} \mathbf{r} f(\mathbf{r}) e^{-i \mathbf{q} \cdot \mathbf{r}}
$$

and

$$
f(\mathbf{r})=\frac{1}{2 \pi} \int d^{2} \mathbf{q} \tilde{f}(\mathbf{q}) e^{i \mathbf{q} \cdot \mathbf{r}}
$$

we have

$$
f(\mathbf{r}) \approx \sum_{\mathbf{q}} \frac{2 \pi}{L_{x} L_{y}} \tilde{f}(\mathbf{q}) e^{i \mathbf{q} \cdot \mathbf{r}}
$$

The Fourier transform of the density operator, $\rho(\mathbf{r}) \equiv$ $\Psi^{\dagger}(\mathbf{r}) \Psi(\mathbf{r})$, is given by

$$
\begin{aligned}
\rho(\mathbf{q}) & =\frac{1}{2 \pi} \int d^{2} \mathbf{r} \rho(\mathbf{r}) e^{-i \mathbf{q} \cdot \mathbf{r}} \\
& =\frac{1}{2 \pi} \int d^{2} \mathbf{r} \sum_{k, k^{\prime}} \psi_{k}^{\dagger}(\mathbf{r}) \psi_{k^{\prime}}(\mathbf{r}) e^{-i \mathbf{q} \cdot \mathbf{r}} a_{k}^{\dagger} a_{k^{\prime}}
\end{aligned}
$$

With the following change of variables

$$
q_{y}=k^{\prime}-k, \quad k_{0}=\frac{k+k^{\prime}}{2}, \quad k_{ \pm}=k_{0} \mp \frac{q_{y}}{2}
$$

the density operator becomes

$$
\begin{aligned}
\rho(\mathbf{q})= & \frac{1}{2 \pi^{3 / 2} l_{0}} \sum_{k_{0}} \int d x \times \\
& e^{-\frac{1}{2 l_{0}^{2}}\left(x+k_{+} l_{0}^{2}\right)^{2}-\frac{1}{2 l_{0}^{2}}\left(x+k_{-} l_{0}^{2}\right)^{2}-i q_{x} x} a_{k_{+}}^{\dagger} a_{k_{-}} \\
= & \frac{1}{2 \pi} \sum_{k_{0}} \exp \left[-\frac{1}{4} q^{2} l_{0}^{2}+i k_{0} q_{x} l_{0}^{2}\right] a_{k_{+}}^{\dagger} a_{k_{-}}
\end{aligned}
$$

Define the interaction operator $\hat{V}$ : 


$$
\begin{aligned}
\hat{V}= & \frac{1}{2} \int d^{2} \mathbf{r} d^{2} \mathbf{r}^{\prime} V\left(\mathbf{r}, \mathbf{r}^{\prime}\right) \rho(\mathbf{r}) \rho\left(\mathbf{r}^{\prime}\right) \\
= & \frac{(2 \pi)^{3}}{2 L_{x} L_{y}} \sum_{\mathbf{q}} \tilde{V}(\mathbf{q}) \rho(-\mathbf{q}) \rho(\mathbf{q}) \\
= & \frac{\pi}{L_{x} L_{y}} \sum_{\mathbf{q}} \sum_{k_{0}, k_{0}^{\prime}} \tilde{V}(\mathbf{q}) \times \\
& \quad \exp \left[-\frac{1}{2} q^{2} l_{0}^{2}+i\left(k_{0}^{\prime}-k_{0}\right) q_{x} l_{0}^{2}\right] a_{k_{+}^{\prime}}^{\dagger} a_{k_{-}^{\prime}} a_{k_{-}}^{\dagger} a_{k_{+}}
\end{aligned}
$$

In the Hartree-Fock (HF) approximation, the energy of the system is given by

$$
\begin{array}{r}
\langle\hat{V}\rangle=\frac{\pi}{L_{x} L_{y}} \sum_{\mathbf{q}, k_{0}, k_{0}^{\prime}} \tilde{V}(\mathbf{q}) e^{-\frac{1}{2} q^{2} l_{0}^{2}+i\left(k_{0}^{\prime}-k_{0}\right) q_{x} l_{0}^{2}} \times \\
{\left[\left\langle a_{k_{+}^{\prime}}^{\dagger} a_{k_{-}^{\prime}}\right\rangle\left\langle a_{k_{-}}^{\dagger} a_{k_{+}}\right\rangle-\left\langle a_{k_{+}^{\prime}}^{\dagger} a_{k_{+}}\right\rangle\left\langle a_{k_{-}}^{\dagger} a_{k_{-}^{\prime}}\right\rangle\right]}
\end{array}
$$

In terms of the operator $\Delta(\mathbf{q}) \equiv \frac{1}{2 \pi} \sum_{k} e^{-i k q_{x} l_{0}^{2}}\left\langle a_{k_{+}}^{\dagger} a_{k_{-}}\right\rangle$, which represents the density of orbit centers, the energy is

$$
\begin{aligned}
\langle\hat{V}\rangle & =\frac{(2 \pi)^{3}}{2 L_{x} L_{y}} \sum_{\mathbf{q}}\left[\tilde{U}(q)-l_{0}^{2} U\left(q l_{0}^{2}\right)\right] \Delta(-\mathbf{q}) \Delta(\mathbf{q}) \\
= & \frac{1}{2} \int d^{2} \mathbf{r} d^{2} \mathbf{r}^{\prime}\left[\begin{array}{c}
\left.U\left(\left|\mathbf{r}-\mathbf{r}^{\prime}\right|\right)-\frac{1}{l_{0}^{2}} \tilde{U}\left(\frac{\left|\mathbf{r}-\mathbf{r}^{\prime}\right|}{l_{0}^{2}}\right)\right] \times \\
\Delta(\mathbf{r}) \Delta\left(\mathbf{r}^{\prime}\right)
\end{array}\right.
\end{aligned}
$$

where $\tilde{U}(q) \equiv V(q) e^{-\frac{1}{2} q^{2} l_{0}^{2}}$ and $U(r)$ is its Fourier transform. It is convenient to define $\tilde{U}_{H F}(q)=\tilde{U}(q)-l_{0}^{2} U\left(q l_{0}^{2}\right)$ where the first term corresponds to the direct interaction and the second to the exchange interaction. $\langle\hat{V}\rangle$ in the above equation gives the CF-CF interaction energy, to which the CF-background and background-background interaction energies must be added to obtain a finite result. Following Ref. ??, we define the cohesive energy as the energy difference measured from the uniform HartreeFock state

$$
\begin{aligned}
E_{c o h}= & \frac{1}{N} \frac{(2 \pi)^{3}}{2 L_{x} L_{y}} \times \\
& \sum_{\mathbf{q} \neq 0}\left[\tilde{U}(q)-l_{0}^{2} U\left(q l_{0}^{2}\right)\right] \Delta(-\mathbf{q}) \Delta(\mathbf{q})
\end{aligned}
$$

We can now calculate the cohesive energies of the stripe and bubble phases. In the stripe phase with the periodic length of stripe $\Lambda$, the orbit-center density is written as

$$
\Delta(x, y)=\frac{1}{2 \pi l_{0}^{2}} \sum_{q} \frac{2 \sin \left(\frac{q \Lambda \nu}{2}\right)}{\Lambda q} e^{i q x}
$$

where $\{q\}=\frac{2 \pi}{\Lambda} \times\{\cdots,-3,-2,-1,1,2,3, \cdots\}$. So the cohesive energy is

$$
E_{c o h}=\frac{1}{2 \nu l_{0}^{2}} \sum_{q} \tilde{U}_{H F}(q)\left(\frac{2 \sin \left(\frac{q \Lambda \nu}{2}\right)}{\Lambda q}\right)^{2}
$$

In the bubble phase with the lattice constant $\Lambda_{b}$ for the hexagonal lattice, radius of the bubble $R=\sqrt{\frac{\sqrt{3} \nu}{2 \pi}} \Lambda_{b}$ and area of a cell $A=\frac{\sqrt{3}}{2} \Lambda_{b}^{2}$, the orbit-center density is

$$
\Delta(\mathbf{r})=\frac{1}{A} \sum_{\mathbf{q}} \frac{R}{l_{0}^{2} q} J_{1}(q R) e^{i \mathbf{q} \cdot \mathbf{r}}
$$

where $\mathbf{q}=n \mathbf{e}_{\mathbf{1}}+m \mathbf{e}_{\mathbf{2}}, \mathbf{e}_{\mathbf{1}}=\frac{4 \pi}{\sqrt{3} \Lambda_{b}} \hat{y}, \mathbf{e}_{\mathbf{2}}=\frac{2 \pi}{\Lambda_{b}} \hat{x}-\frac{2 \pi}{\sqrt{3} \Lambda_{b}} \hat{y}$. This gives the cohesive energy

$$
E_{c o h}=\frac{4 \pi}{\sqrt{3} l_{0}^{2} \Lambda_{b}^{2}} \sum_{\mathbf{q}} \tilde{U}_{H F}(q)\left(\frac{R}{A l_{0}^{2} q} J_{1}(q R)\right)^{2} .
$$

With our effective interaction the sums in stripe and bubble calculations converge quickly as we increase the cut off for q. Ref. [24], which deals with electrons in the higher electronic LLs, finds that the transition from the bubble to the stripe phase occurs at $\nu_{n} \approx 0.4$, where $\nu_{n}$ is the electron filling in the $(n+1)^{s t}$ Landau level. Somewhat surprisingly, we also find a transition at $\nu_{n}^{*} \approx$ 0.4 in all of our calculations.

As far as the comparison of stripe and bubble phases is concerned, it is sufficient to know the cohesive energy; the energy of the reference, uniform HF state need not be evaluated. However, to compare with the Fermi sea, the FQHE state, or the paired state, we also need to know the energy of the uniform HF state, which must be subtracted from the full energy to obtain the cohesive energy.

To calculate the energy of the uniform HF state we assume that the CF-CF, background-background, and CFbackground interactions are all of the same form, characterized by $V_{m}^{C F}$. Since the direct interaction terms cancel each other the only remaining part is the exchange term of the CF-CF interaction. Thus the energy of the uniform state $E_{0}$ is

$$
E_{0}=-\frac{U(0)}{2} \times \nu
$$

For example in the case of the Coulomb interaction:

$$
E_{0}=-\frac{U(0)}{2} \times \nu=-\frac{1}{2} \int_{0}^{\infty} d q e^{\frac{1}{2} q^{2}} \times \nu=-\sqrt{\frac{\pi}{8}} \times \nu
$$

which is a familiar result. For other interactions, we evaluate $E_{0}$ numerically.

\section{RESULTS AND DISCUSSION}

The energies of various states are shown in Fig. 2 of Ref. [17], and Figs. (2) and (3) of the present manuscript. The lowest energy for the stripe or bubble states are determined by varying the period. We draw the following conclusions (subject to the validity of the model): 
(i) FQHE: Within the lowest ${ }^{2} \mathrm{CF}$ Landau level, CF holes capture two more vortices to become ${ }^{4} \mathrm{CFs}$. They show quantum Hall effect at $\nu_{n}^{*}=1 / 3$, which corresponds to ${ }^{2} \mathrm{CF}$ filling factor of $\nu^{*}=1-\frac{1}{3}$, and electron filling factor of $\nu=\frac{2}{7}$. At $\nu_{n}^{*}=1 / 2$, which corresponds to $\nu=1 / 4$, the Fermi sea has the lowest energy. These results are consistent with the observations of FQHE at $2 / 7$ and CF Fermi sea at $1 / 4$.

(ii) Stripes: At $\nu^{*}=n+\frac{1}{2}$, which correspond to $\nu=(2 n+1) /[2(2 n+2)]$, the stripe phase has the lowest energy (for $n \geq 1$ ). The stripe phase is obtained independently of whether we model $\nu^{*}=n+\frac{1}{2}$ as $1 / 2$ filling of CF particles on the background of $n$ filled ${ }^{2} \mathrm{CF}$ Landau levels, or as $1 / 2$ filling of $\mathrm{CF}$ holes on the background of $n+1$ filled ${ }^{2} \mathrm{CF}$ Landau levels, which suggests that the result is robust. It should be noted, however, that the issues regarding the stability of the unidirectional charge density wave against a modulation along the length and also against quantum fluctuations, which have been investigated in the context of electronic stripes in higher Landau levels [26], have not been considered here.

The lowest energy for stripes is obtained for period $\Lambda / l_{0}=10,28$, and 34 for $\nu=3 / 8,5 / 12$, and $7 / 16$. The period is rather large compared to that for the electron stripes in higher LLs (for which $\Lambda / l_{0}$ is of order unity), which is not surprising because the interaction between composite fermions is rather weak, and also the difference between the densities of the FQHE states on either side is quite small.

A transport anisotropy in higher electronic Landau levels, interpreted in terms of stripe formation, is observed at temperatures below $\sim 50 \mathrm{mK}$ [27]. The conditions for the $\mathrm{CF}$ stripes are more stringent. Estimates of the critical temperature from the Hartree Fock theory are not quantitatively reliable, but noting that the effective interaction between composite fermions at $\nu^{*}=n+1 / 2$ is roughly an order of magnitude smaller than for electrons at $\nu=n+1 / 2$, as measured by the pseudopotentials, we expect the critical temperature to also be similarly reduced. Also, the much larger period suggests the need for a high degree of density homogeneity.

(iii) Bubbles: At $\nu^{*}=n \pm \frac{1}{3}$ with $n \geq 1$, the bubble phase has the lowest energy. We remind the reader, however, that our trial wave functions for FQHE states that work very well in the lowest Landau level are not so good in higher Landau levels, which makes our calculation somewhat biased against FQHE.

To estimate at what filling a transition occurs from the bubble crystal to the stripe phase, we have determined their energies as a function of the filling factor, shown in Fig. (4). The stripes are found to be stable approximately in the region $0.4<\nu^{*}<0.6$, outside of which bubbles take over. Overall, the phase diagram for various states of composite fermions, shown schematically in Fig. 5, is remarkably similar to that for electrons. For electrons: (i) FQHE occurs in the lowest Landau level; (ii) stripes are believed to be relevant in the vicinity of $\nu=n+1 / 2$ for $n \geq 2$; (iii) bubble or Wigner crystal takes over in higher Landau levels for $\nu=n+\nu^{\prime}$ with $\nu^{\prime}<0.4$. The behavior for ${ }^{2} \mathrm{CFs}$ is quite analogous.

\section{FINITE THICKNESS}

We have assumed until now that the electron layer width is zero. In actual experiments, the electron wave function has a finite extent in the transverse direction, which modifies the interaction pseudopotentials. The modified interaction has been obtained in a local-density approximation $[28,29]$. To estimate how finite thickness affects the results presented above, we have calculated the pseudopotentials for composite fermions in the second CF-LL and found (Fig. 6) that the value $V_{3}^{C F}$, which is the largest pseudopotential, is reduced as we increase the density of the electrons. However, the change is not large enough to alter the previous results. As seen in Fig. (7), at $\nu=4 / 11$, where the composite fermion filling is $\nu^{*}=4 / 3$, the bubble crystal phase continues to be most favorable among the ones studied.

\section{CONCLUSION}

In summary, we have considered theoretically the question of what new states of composite fermions are feasible as a result of the residual interaction between composite fermions. For this purpose, we have constructed a model for the CF-CF interaction, and studied various plausible states within a variational scheme. Our results suggest that fractional quantum Hall effect, stripe phase, as well as bubble crystal of composite fermions can all occur at various filling factors.

\section{Acknowledgements}

This work was supported in part by the National Science Foundation under grants no. DMR-9986806 and DGE-9987589. We are grateful to the High Performance Computing (HPC) Group led by V. Agarwala, J. Holmes, and J. Nucciarone, at the Penn State University ASET (Academic Services and Emerging Technologies) for assistance and computing time with the LION-XE cluster.

\section{Appendix}

One of the most time consuming aspects of our Monte Carlo calculation is the evaluation of determinants. When two matrices have many common columns (rows), the determinant of one can be related to the other, which amounts to substantial saving of computation time in Monte Carlo. Here we describe a general method for it.

Consider two $n \times n$ matrices $\mathbf{A}$ and $\mathbf{A}^{\prime}$, which differ only in the $\left(i_{1}, i_{2}, \cdots, i_{m}\right)$ th columns, where $0<m \leq$ 
$n$. Our aim is to obtain the determinant of $\mathbf{A}^{\prime}$ with minimum computation. The determinant of $\mathbf{A}^{\prime}$ can be written as

$$
\operatorname{det}\left[\mathbf{A}^{\prime}\right]=\operatorname{det}[\mathbf{A}] \operatorname{det}\left[\mathbf{A}^{-1} \mathbf{A}^{\prime}\right]
$$

Since $A^{\prime}$ differs from $A$ only at $\left(i_{1}, i_{2}, \cdots, i_{m}\right)$ th columns, $\left[\mathbf{A}^{-\mathbf{1}} \mathbf{A}^{\prime}\right]_{k l}$ is equal to $\delta_{k l}$ when $l \notin\left(i_{1}, i_{2}, \cdots, i_{m}\right)$

Define $\mathbf{C}_{j}(j=1,2, \cdots, m)$ to be $n \times n$ matrices whose components are same as those of $\mathbf{A}$ at $j$ th column and zero elsewhere. Further, define an $m \times m$ matrix $\mathbf{B}$ such that $[\mathbf{B}]_{a b}=\left[\mathbf{A}^{-\mathbf{1}} \mathbf{C}_{\mathbf{b}}^{\prime}\right]_{i_{a} i_{b}}$ wher e $a, b \in\{1,2, \cdots, m\}$. Then the following is true.

$$
\operatorname{det}\left[\mathbf{A}^{-\mathbf{1}} \mathbf{A}^{\prime}\right]=\operatorname{det}[\mathbf{B}]
$$

This gives a simple relation between the determinants of $\mathbf{A}^{\prime}$ and $\mathbf{A}$ :

$$
\operatorname{det}\left[\mathbf{A}^{\prime}\right]=\operatorname{det}[\mathbf{A}] \operatorname{det}[\mathbf{B}]
$$

Consider some examples:

1. Updating of one column:

$$
\operatorname{det}\left[\mathbf{A}^{\prime}\right]=\operatorname{det}[\mathbf{A}] \operatorname{det}[\mathbf{B}]=\operatorname{det}[\mathbf{A}]\left[\mathbf{A}^{-\mathbf{1}} \mathbf{C}_{\mathbf{1}}^{\prime}\right]_{i_{1} i_{1}}
$$

2. Updating of two columns:

$$
\begin{aligned}
& \operatorname{det}\left[\mathbf{A}^{\prime}\right]= \operatorname{det}[\mathbf{A}] \operatorname{det}[\mathbf{B}] \\
&=\operatorname{det}[\mathbf{A}]\left(\left[\mathbf{A}^{-\mathbf{1}} \mathbf{C}_{\mathbf{1}}^{\prime}\right]_{i_{1} i_{1}}\left[\mathbf{A}^{-\mathbf{1}} \mathbf{C}_{\mathbf{2}}^{\prime}\right]_{i_{2} i_{2}}\right. \\
&\left.-\left[\mathbf{A}^{-\mathbf{1}} \mathbf{C}_{\mathbf{1}}^{\prime}\right]_{i_{2} i_{1}}\left[\mathbf{A}^{-\mathbf{1}} \mathbf{C}_{\mathbf{2}}^{\prime}\right]_{i_{1} i_{2}}\right)
\end{aligned}
$$

3. Updating of three columns:

$$
\begin{aligned}
\operatorname{det}\left[\mathbf{A}^{\prime}\right]= & \operatorname{det}[\mathbf{A}] \operatorname{det}[\mathbf{B}] \\
= & \operatorname{det}[\mathbf{A}] \times \\
& \left(\left[\mathbf{A}^{-\mathbf{1}} \mathbf{C}_{\mathbf{1}}^{\prime}\right]_{i_{1} i_{1}}\left[\mathbf{A}^{-\mathbf{1}} \mathbf{C}_{\mathbf{2}}^{\prime}\right]_{i_{2} i_{2}}\left[\mathbf{A}^{-\mathbf{1}} \mathbf{C}_{\mathbf{3}}^{\prime}\right]_{i_{3} i_{3}}\right. \\
& +\left[\mathbf{A}^{-\mathbf{1}} \mathbf{C}_{\mathbf{1}}^{\prime}\right]_{i_{2} i_{1}}\left[\mathbf{A}^{-\mathbf{1}} \mathbf{C}_{\mathbf{2}}^{\prime}\right]_{i_{3} i_{2}}\left[\mathbf{A}^{-\mathbf{1}} \mathbf{C}_{\mathbf{3}}^{\prime}\right]_{i_{1} i_{3}} \\
& +\left[\mathbf{A}^{-\mathbf{1}} \mathbf{C}_{\mathbf{1}}^{\prime}\right]_{i_{3} i_{1}}\left[\mathbf{A}^{-\mathbf{1}} \mathbf{C}_{\mathbf{2}}^{\prime}\right]_{i_{1} i_{2}}\left[\mathbf{A}^{-\mathbf{1}} \mathbf{C}_{\mathbf{3}}^{\prime}\right]_{i_{2} i_{3}} \\
& {\left[\mathbf{A}^{-\mathbf{1}} \mathbf{C}_{\mathbf{1}}^{\prime}\right]_{i_{2} i_{1}}\left[\mathbf{A}^{-\mathbf{1}} \mathbf{C}_{\mathbf{2}}^{\prime}\right]_{i_{1} i_{2}}\left[\mathbf{A}^{-\mathbf{1}} \mathbf{C}_{\mathbf{3}}^{\prime}\right]_{i_{3} i_{3}} } \\
& -\left[\mathbf{A}^{-\mathbf{1}} \mathbf{C}_{\mathbf{1}}^{\prime}\right]_{i_{3} i_{1}}\left[\mathbf{A}^{-\mathbf{1}} \mathbf{C}_{\mathbf{2}}^{\prime}\right]_{i_{2} i_{2}}\left[\mathbf{A}^{-\mathbf{1}} \mathbf{C}_{\mathbf{3}}^{\prime}\right]_{i_{1} i_{3}} \\
& \left.-\left[\mathbf{A}^{-\mathbf{1}} \mathbf{C}_{\mathbf{1}}^{\prime}\right]_{i_{1} i_{1}}\left[\mathbf{A}^{-\mathbf{1}} \mathbf{C}_{\mathbf{2}}^{\prime}\right]_{i_{3} i_{2}}\left[\mathbf{A}^{-\mathbf{1}} \mathbf{C}_{\mathbf{3}}^{\prime}\right]_{i_{2} i_{3}}\right)
\end{aligned}
$$

[1] J.K. Jain, Phys. Rev. Lett. 63199 (1989); Phys. Rev. B 41, 7653 (1990); Physics Today, 53(4), 39 (2000).

[2] Composite Fermions, edited by Olle Heinonen (World Scientific, New York, 1998); Perspectives in Quantum Hall Effects, edited by S. Das Sarma and A. Pinczuk (Wiley, New York, 1997).
[3] D.C. Tsui, H.L. Stormer, and A.C. Gossard, Phys. Rev. Lett. 48, 1559 (1982).

[4] K. v. Klitzing, G. Dorda, and M. Pepper, Phys. Rev. Lett. 45, 494 (1980).

[5] B.I. Halperin, P.A. Lee, and N. Read, Phys. Rev. B 47, 7312 (1993); V.Kalmeyer and S.-C. Zhang, Phys. Rev. B 46, 9889 (1992).

[6] R. L. Willett, R. R. Ruel, K. W. West, and L. N. Pfeiffer, Phys Rev. Lett. 71, 3846 (1993); W. Kang, H. L. Stormer, L. N. Pfeiffer, K. W. Baldwin, and K. W. West , Phys. Rev. Lett. 71, 3850 (1993); V.J. Goldman, B. Su, J.K. Jain, Phys Rev. Lett. 72, 2065 (1994).

[7] G. Dev and J. K. Jain, Phys. Rev. Lett. 69, 2843 (1992); X.G. Wu, G. Dev, J.K. Jain, Phys. Rev. Lett. 71, 153 (1993).

[8] J. K. Jain and R. K. Kamilla, Int. J. Mod. Phys. B 11, 2621 (1997); Phys. Rev. B 55, R4895 (1997).

[9] R.K. Kamilla and J.K. Jain, Phys. Rev. B 5513417 (1997).

[10] V.W. Scarola, K. Park and J.K. Jain, Phys. Rev. B 62, R16259 (2000).

[11] W. Pan, H.L. Stormer, D.C. Tsui, L.N. Pfeiffer, K.W. Baldwin, and K.W. West, Phys. Rev. Lett. 88, 176802 (2002).

[12] V. W. Scarola, K. Park, and J. K. Jain, Nature 406, 863 (2000).

[13] G. Moore and N. Read, Nucl. Phys. B 360, 362 (1991); M. Greiter, X.G. Wen, and F. Wilczek, Phys. Rev. Lett. 66, 3205 (1991); R.H. Morf, Phys. Rev. Lett. 80, 1505 (1998); E.H. Rezayi and F.D.M. Haldane, Phys. Rev. Lett. 84, 4685 (2000).

[14] R.L. Willett, J. P. Eisenstein, H. L. St?rmer, D. C. Tsui, A. C. Gossard, and J. H. English, Phys. Rev. Lett. 59, 1776 (1987); W. Pan, J.-S. Xia, V. Shvarts, D. E. Adams, H. L. Stormer, D. C. Tsui, L. N. Pfeiffer, K. W. Baldwin, and K. W. West, Phys. Rev. Lett. 83, 3530 (1999).

[15] K. Park and J.K. Jain, Phys. Rev. B 62, R13274 (2000).

[16] V.W. Scarola, J.K. Jain, and E.H. Rezayi, Phys. Rev. Lett. 88, 216804 (2002).

[17] S.-Y. Lee, V.W. Scarola, and J.K. Jain, Phys. Rev. Lett. 87, 256803 (2001).

[18] A. Wojs and J. J. Quinn, Phys. Rev. B 61, 2846 (2000).

[19] F. D. M. Haldane, Phys. Rev. Lett. 51, 605 (1983).

[20] J.J. Quinn and A. Wojs, J. Phys. Cond. Mat. 12, R265 (2000), and references therein.

[21] K. Park and J.K. Jain, Phys. Rev. Lett. 84, 5576 (2000).

[22] D. Ceperley, G.V. Chester, and M.H. Kalos, Phys. Rev. B16, 3081 (1977).

[23] K. Park, V. Melik-Alaverdian, N. E. Bonesteel, and J.K. Jain, Phys. Rev. B 58, R10167 (1998).

[24] M. M. Fogler, A. A. Koulakov, and B. I. Shklovskii, Phys. Rev. B 54, 1853 (1996).

[25] Charge density wave and stripe phase for electrons in the lowest LL have also been considered. See Y. Kuramoto, J. Phys. Soc. Jpn. 45, 390 (1978); D. Yoshioka and P.A. Lee, Phys. Rev. B27, 4986 (1983).

[26] E. Fradkin and S.A. Kivelson, Phys. Rev. B59, 8065 (1999); H. Yi, H.A. Fertig, and R. Côté, Phys. Rev. Lett. 85, 4156 (2000).

[27] M.P. Lilly K. B. Cooper, J. P. Eisenstein, L. N. Pfeiffer, and K. W. West, Phys. Rev. Lett. 82, 394 (1999); 
R.R. Du, D.C. Tsui, H.L. Stormer, L.N. Pfeiffer, K.W. Baldwin and K.W. West, Solid State Commun. 109, 389 (1999).

[28] M. W. Ortalano, S. He and S. Das Sarma, Phys. Rev. B 55, 7702 (1997).

[29] K. Park, N. Meskini, and J. K. Jain, J. Phys. Condens. Matter. 11, 7283 (1999).
TABLE I. Pseudopotentials for CF-holes in the lowest CF Landau level. In this and the following tables, $E_{m}$ is the energy of the full state, and $\Delta E_{m}$ is the Monte Carlo statistical uncertainty. The energies are quoted in units of $e^{2} / \epsilon l_{0}$.

\begin{tabular}{ccc}
\hline \hline$m$ & $E_{m}$ & $\Delta E_{m}$ \\
\hline 1 & -7.726983 & 0.0005744120 \\
3 & -7.757470 & 0.0007778533 \\
5 & -7.752651 & 0.0010310604 \\
7 & -7.758716 & 0.0010988030 \\
9 & -7.758750 & 0.0012034221 \\
11 & -7.759422 & 0.0012985310 \\
13 & -7.760764 & 0.0012624134 \\
15 & -7.761201 & 0.0012929297 \\
17 & -7.762082 & 0.0016546843 \\
19 & -7.762933 & 0.0016976433 \\
\hline \hline
\end{tabular}

TABLE II. Pseudopotentials for CF-particles in the 2nd CF Landau level.

\begin{tabular}{ccc}
\hline \hline$m$ & $E_{m}$ & $\Delta E_{m}$ \\
\hline 1 & -11.755883 & 0.0005593626 \\
3 & -11.741289 & 0.0006092897 \\
5 & -11.756699 & 0.0005888317 \\
7 & -11.754222 & 0.0005821469 \\
9 & -11.754109 & 0.0006913264 \\
11 & -11.755223 & 0.0006993560 \\
13 & -11.755586 & 0.0008570091 \\
15 & -11.755554 & 0.0005545685 \\
17 & -11.756373 & 0.0006021690 \\
19 & -11.756839 & 0.0005796026 \\
21 & -11.756438 & 0.0005914016 \\
23 & -11.756528 & 0.0006283216 \\
25 & -11.756233 & 0.0006583812 \\
27 & -11.756788 & 0.0006599042 \\
\hline \hline
\end{tabular}

TABLE III. Pseudopotentials for CF-holes in the 2nd CF Landau level.

\begin{tabular}{ccc}
\hline \hline$m$ & $E_{m}$ & $\Delta E_{m}$ \\
\hline 1 & -18.135544 & 0.0009774497 \\
3 & -18.120577 & 0.0009665822 \\
5 & -18.133069 & 0.0009376358 \\
7 & -18.131464 & 0.0008402889 \\
9 & -18.130383 & 0.0009074839 \\
11 & -18.130536 & 0.0009764340 \\
13 & -18.131314 & 0.0009651683 \\
15 & -18.130754 & 0.0010156039 \\
17 & -18.131685 & 0.0009203913 \\
19 & -18.130155 & 0.0009442089 \\
21 & -18.131228 & 0.0010169967 \\
\hline \hline
\end{tabular}


TABLE IV. Pseudopotentials for CF-particles in the 3rd CF Landau level.

\begin{tabular}{ccc}
\hline \hline$m$ & $E_{m}$ & $\Delta E_{m}$ \\
\hline 1 & -18.942399 & 0.0010950836 \\
3 & -18.932547 & 0.0012154270 \\
5 & -18.927645 & 0.0011055870 \\
7 & -18.935310 & 0.0012656451 \\
9 & -18.934032 & 0.001233024 \\
11 & -18.934417 & 0.0012795043 \\
13 & -18.933864 & 0.0013178322 \\
15 & -18.933060 & 0.0012459279 \\
17 & -18.935017 & 0.0013152929 \\
19 & -18.934135 & 0.0013808266 \\
21 & -18.934547 & 0.0015265486 \\
23 & -18.934336 & 0.0012488644 \\
\hline \hline
\end{tabular}

TABLE V. Pseudopotentials for CF-holes in the 3rd CF Landau level.

\begin{tabular}{ccc}
\hline \hline$m$ & $E_{m}$ & $\Delta E_{m}$ \\
\hline 1 & -17.620082 & 0.000983051 \\
3 & -17.612904 & 0.000917553 \\
5 & -17.606845 & 0.001063009 \\
7 & -17.614824 & 0.001009564 \\
9 & -17.615345 & 0.000978099 \\
11 & -17.614743 & 0.000939712 \\
13 & -17.614587 & 0.000958717 \\
15 & -17.612469 & 0.000891311 \\
\hline \hline
\end{tabular}

TABLE VI. Pseudopotentials for CF-particles in the 4th CF Landau level.

\begin{tabular}{ccc}
\hline \hline$m$ & $E_{m}$ & $\Delta E_{m}$ \\
\hline 1 & -16.691824 & 0.00101485 \\
3 & -16.686846 & 0.00105839 \\
5 & -16.682793 & 0.00107136 \\
7 & -16.680260 & 0.00108352 \\
9 & -16.687565 & 0.00107323 \\
11 & -16.684489 & 0.00111337 \\
13 & -16.685492 & 0.00125616 \\
15 & -16.685188 & 0.00095845 \\
\hline \hline
\end{tabular}

TABLE VII. Parameters for our model interaction for two CF particles in an otherwise empty CF-LL. The second, third, and fourth CF-LLs are considered. All of the $c_{i}$ 's not shown here are set equal to zero.

\begin{tabular}{|c|c|c|c|}
\hline \hline parameter & 2nd CF-LL & 3rd CF-LL & 4th CF-LL \\
\hline$c_{2}$ & -1.57357 & 1.75418 & -10.6790 \\
$c_{6}$ & 0.00817294 & -0.0114760 & 0.0793665 \\
$c_{10}$ & $-1.72040 \mathrm{E}-6$ & $2.62965 \mathrm{E}-6$ & $-2.49751 \mathrm{E}-5$ \\
$c_{14}$ & $4.31056 \mathrm{E}-11$ & $-6.30827 \mathrm{E}-11$ & $9.87564 \mathrm{E}-10$ \\
$c_{18}$ & $-1.80465 \mathrm{E}-16$ & $1.96255 \mathrm{E}-16$ & $-7.12056 \mathrm{E}-15$ \\
$c_{22}$ & 0.0 & 0.0 & $9.29168 \mathrm{E}-21$ \\
\hline \hline
\end{tabular}

TABLE VIII. Parameters for our model interaction for two $\mathrm{CF}$ holes in an otherwise full CF-LL. The first, second and third CF-LLs are considered. All of the $c_{i}$ 's not shown here are set equal to zero.

\begin{tabular}{|c|c|c|c|}
\hline \hline parameter & 1st CF-LL & 2nd CF-LL & 3rd CF-LL \\
\hline$c_{2}$ & 2.08604 & -0.641674 & 1.15620 \\
$c_{6}$ & -0.0122001 & 0.00240019 & -0.00699306 \\
$c_{10}$ & $2.85410 \mathrm{E}-6$ & $-2.15587 \mathrm{E}-7$ & $1.38513 \mathrm{E}-6$ \\
$c_{14}$ & $-7.63371 \mathrm{E}-11$ & $-1.27142 \mathrm{E}-12$ & $-2.49865 \mathrm{E}-11$ \\
$c_{18}$ & $2.90620 \mathrm{E}-16$ & $1.48118 \mathrm{E}-17$ & $4.72082 \mathrm{E}-17$ \\
\hline \hline
\end{tabular}




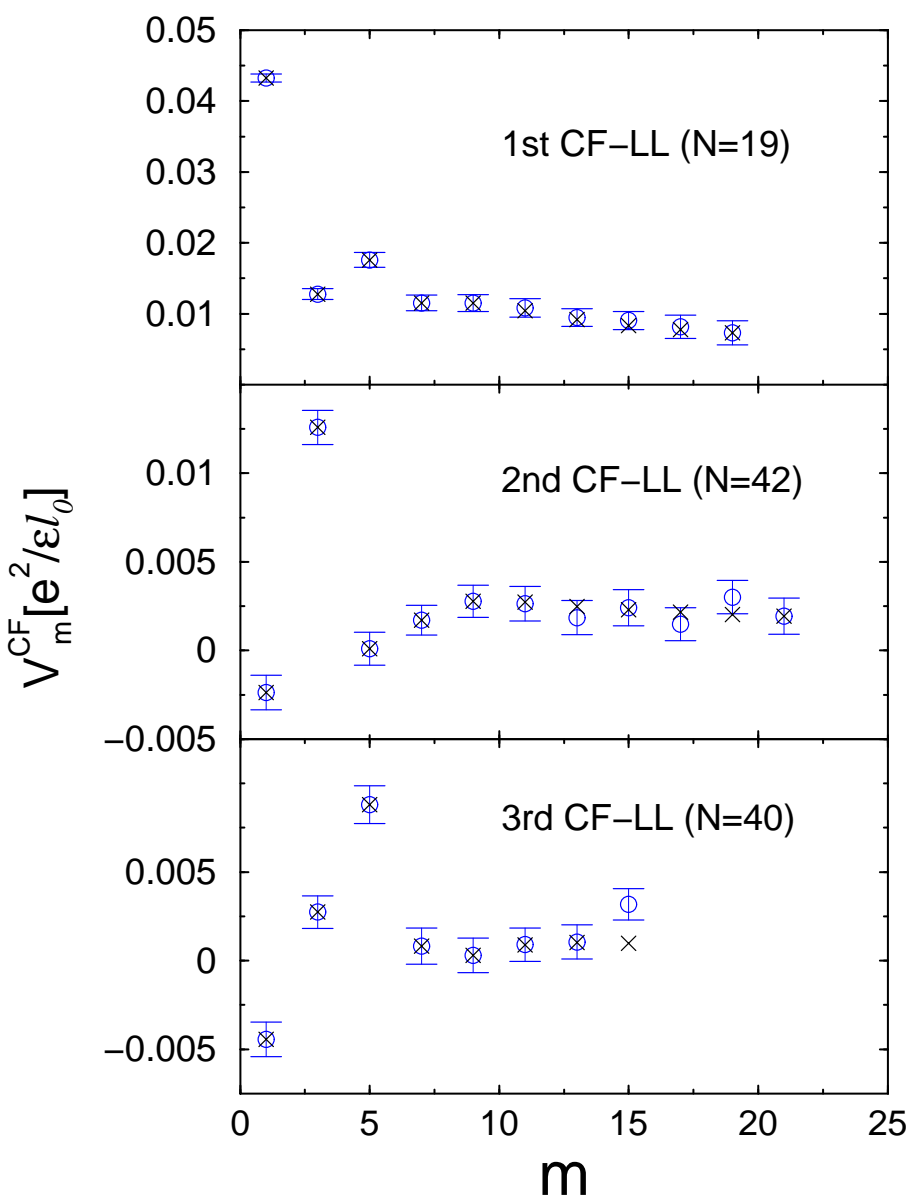

FIG. 1. This figure gives the pseudopotentials for composite fermion "holes" in the first, second, and third composite fermion levels. These are defined (up to an overall additive constant) as the energies of two composite fermion holes in the appropriate composite fermion Landau level, with the lower CF levels fully occupied. The systems are large enough that the pseudopotentials at the first few $m$ (relative angular momentum) values are well converged. Circles denote the pseudopotentials computed from the microscopic wave functions, with error bars showing the Monte Carlo uncertainty, and crosses are the pseudopotentials for the real space model interaction explained in the text. The pseudopotentials for $\mathrm{CF}$ "particles" in the second, third, and fourth composite fermion levels were given in Ref. [17].

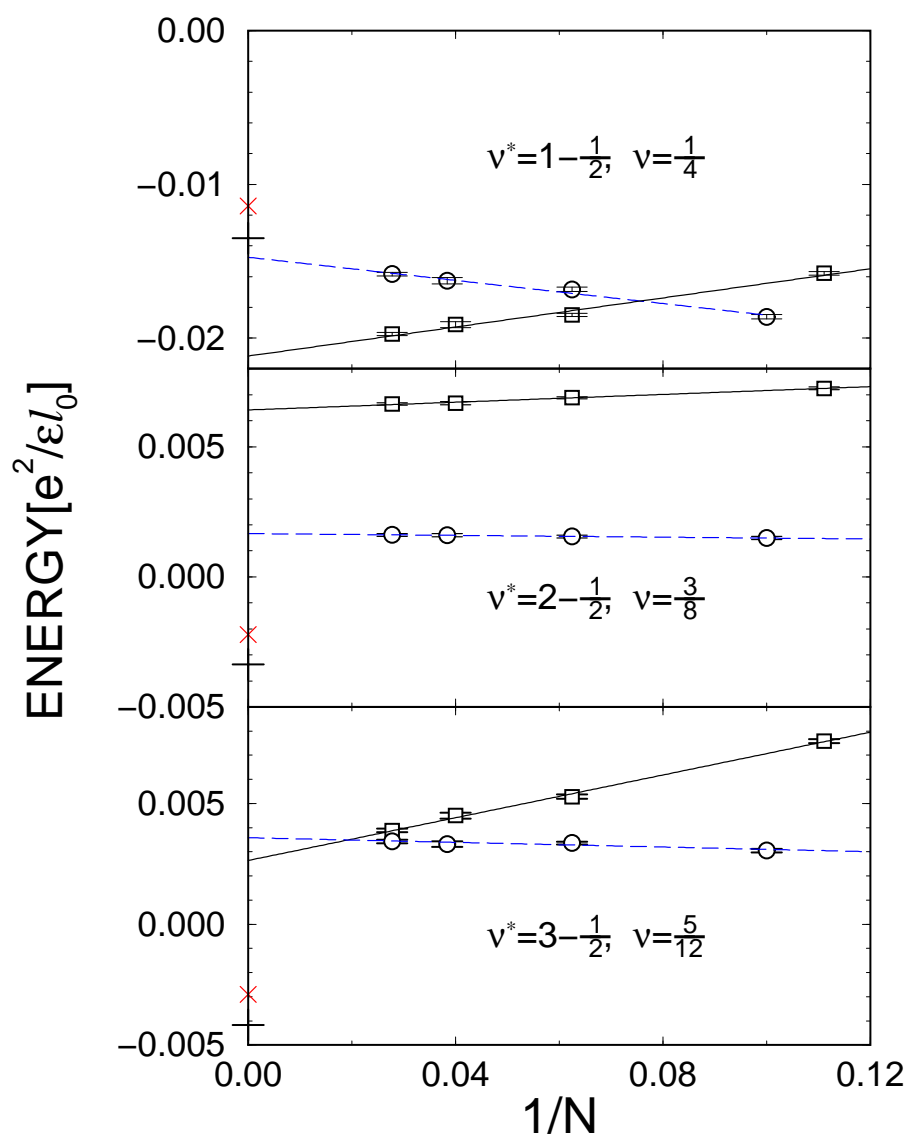

FIG. 2. The cohesive energy per particle for the CF Fermi sea (squares) and the CF paired state (circles) as a function of $N$, the number of CF "holes" in the topmost CF level. The thermodynamic energies are also shown for the CF stripe and bubble phases by a dash $(-)$ and cross $(x)$ on the $y$-axis, respectively. All energies are measured relative to the uncorrelated uniform density state, explained in the text. The corresponding results for CF "particles" at $\nu^{*}=1+1 / 2$, $\nu^{*}=2+1 / 2$, and $\nu^{*}=3+1 / 2$, corresponding to $\nu=3 / 8$, $5 / 12$, and $7 / 16$, were given in Ref. [17]. 


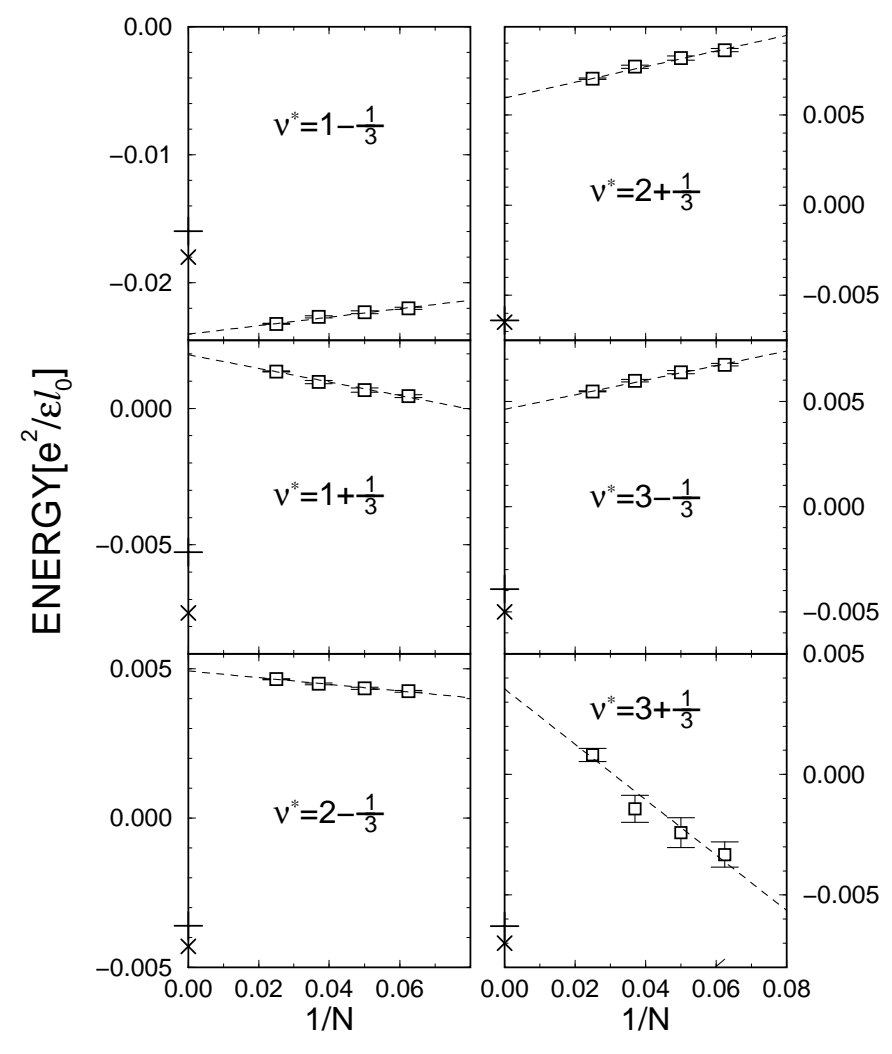

FIG. 3. The cohesive energy per particle for composite fermions at $\nu^{*}=n \pm 1 / 3$, which correspond to electron filling factors $\nu=\frac{3 n \pm 1}{6 n+3 \pm 2}$, for the FQHE state as a function of $N$, the number of composite fermions in the partially filled composite fermion Landau level. This energy is shown by squares. The dash $(-)$ and cross $(x)$ on the $y$-axis show the cohesive energies of the stripe and bubble phases, respectively.

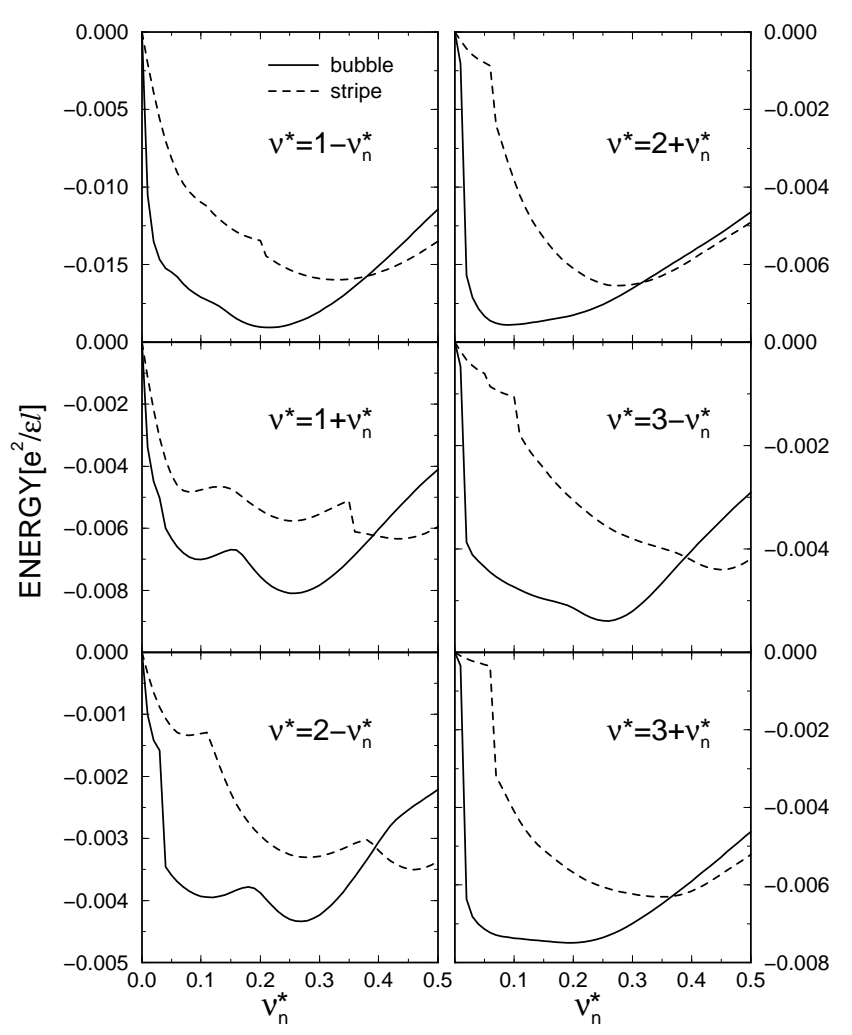

FIG. 4. The cohesive energies of stripes (dashed line) and bubbles (solid line) as a function of the CF filling factor in various CF Landau levels. The striped state has lower energy in the vicinity of $\nu^{*}=n+1 / 2$. 

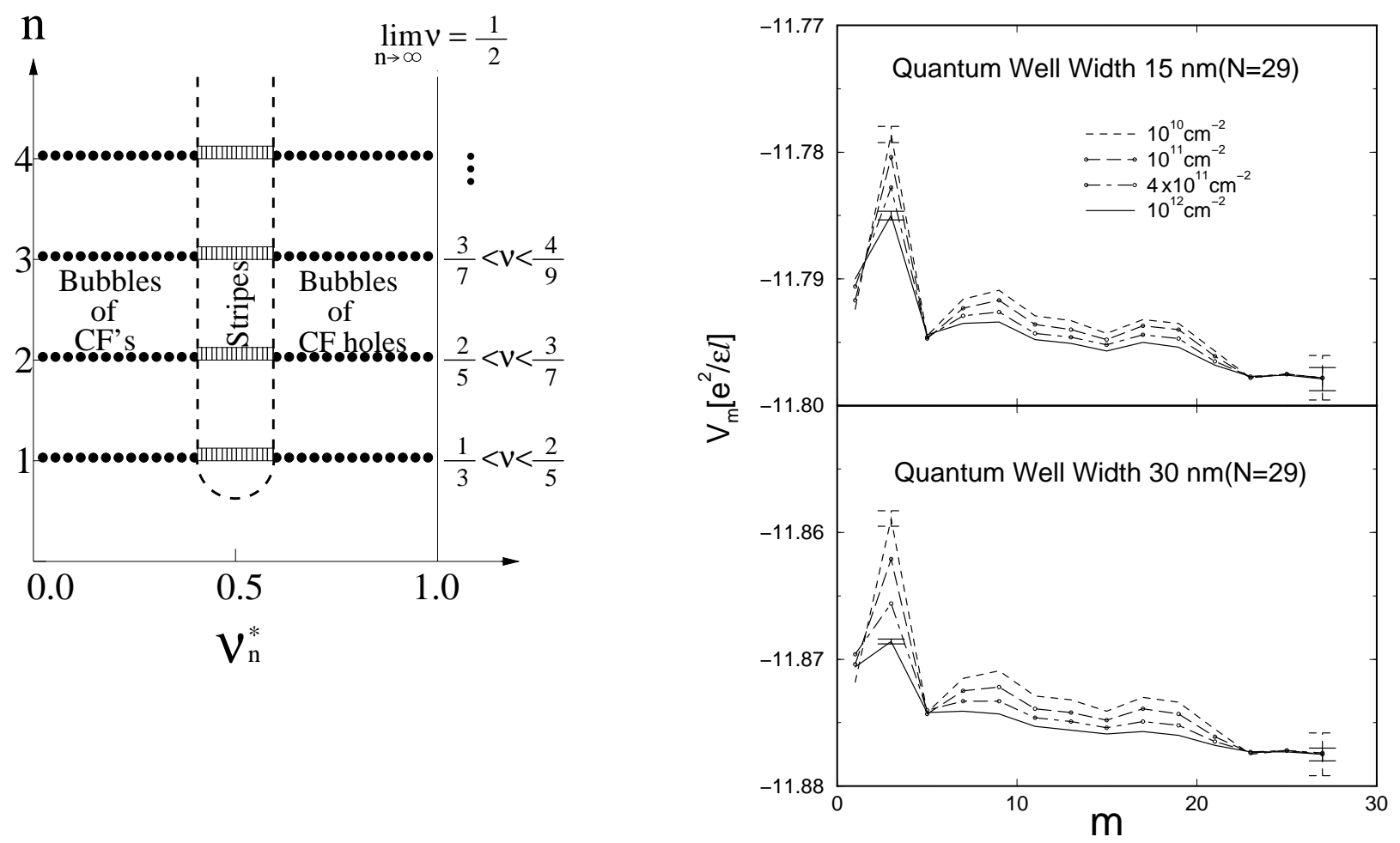

FIG. 5. Phase diagram of various CF states, with bubbles (solid dots), CF stripes (shaded region) and CF FQHE (solid line) as a function of the CF filling $\nu^{*}=n+\nu_{n}^{*}$.

FIG. 6. Pseudopotentials for the CF-CF interaction in the 2nd CF-LL for various densities for quantum wells of widths 15 and $30 \mathrm{~nm}$. The inter-electron interaction has been obtained in a local-density approximation. 


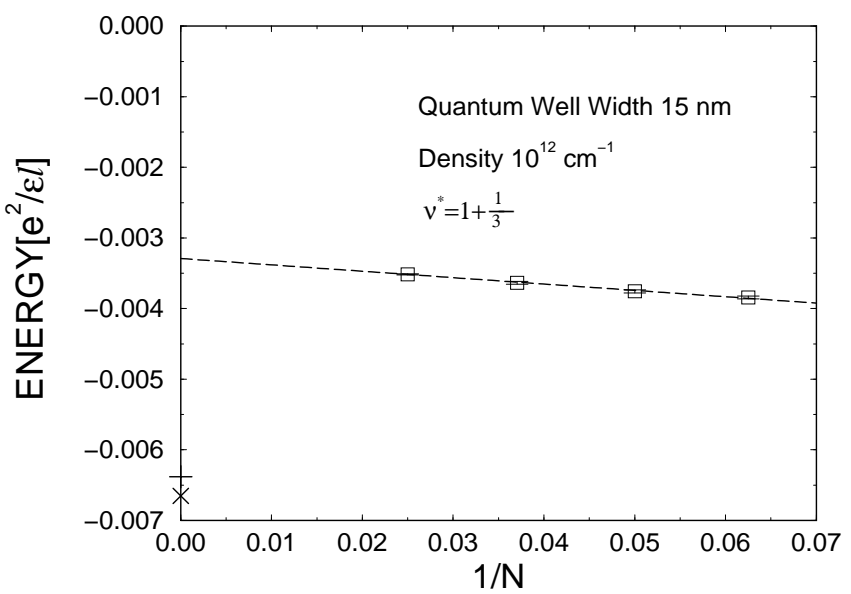

FIG. 7. Same as in Fig. (3) for the modified interaction. 